\title{
Endovascular steerable and endobronchial precurved guiding sheaths for transbronchial needle delivery under augmented fluoroscopy and cone beam CT image guidance
}

\author{
Quirina M. B. de Ruiter ${ }^{1} \wedge$, Joseph R. Fontana ${ }^{2}$, William F. Pritchard ${ }^{1}$, Michal Mauda-Havakuk ${ }^{1}$, \\ Ivane Bakhutashvili ${ }^{1}$, Juan A. Esparza-Trujillo ${ }^{1}$, Nicole A. Varble ${ }^{1,3}$, Marco Verstege ${ }^{4}$, Sheng Xu ${ }^{1}$, \\ Reza Seifabadi ${ }^{1}$, Robert F. Browning ${ }^{5}$, Bradford J. Wood ${ }^{1,6}$, John W. Karanian ${ }^{1}$
}

${ }^{1}$ Center for Interventional Oncology, Radiology \& Imaging Sciences, National Institutes of Health Clinical Center, Bethesda, MD, USA; ${ }^{2}$ Pulmonary Medicine Branch, National Heart, Lung, and Blood Institute, National Institutes of Health, Bethesda, MD, USA; ${ }^{3}$ Philips Research of North America, Cambridge, MA, USA; ${ }^{4}$ Philips, Best, The Netherlands; ${ }^{5}$ Walter Reed National Military Medical Center, Bethesda, MD, USA; ${ }^{6}$ Center for Cancer Research, National Institutes of Health Clinical Center, Bethesda, MD, USA

Contributions: (I) Conception and design: QMB de Ruiter, JR Fontana, WF Pritchard, NA Varble, S Xu, RF Browning, BJ Wood, JW Karanian; (II) Administrative support: QMB de Ruiter, I Bakhutashvili, NA Varble, M Verstege, JW Karanian; (III) Provision of study materials or patients: QMB de Ruiter, I Bakhutashvili, JA Esparza-Trujillo, M Verstege, R Seifabadi, S Xu, BJ Wood, JW Karanian; (IV) Collection and assembly of data: QMB de Ruiter, JR Fontana, WF Pritchard, M Mauda-Havakuk, I Bakhutashvili, JA Esparza-Trujillo, NA Varble, M Verstege, RF Browning, BJ Wood, JW Karanian; (V) Data analysis and interpretation: QMB de Ruiter, M Mauda-Havakuk, Browning, BJ Wood, JW Karanian; (VI) Manuscript writing: All authors; (VII) Final approval of manuscript: All authors.

Correspondence to: John W. Karanian, PhD. Center for Interventional Oncology, Radiology \& Imaging Sciences, National Institutes of Health Clinical Center, MSC 1182, Bldg. 10, Room 3N320C, Bethesda, MD 20892-1182, USA. Email: john.karanian@nih.gov.

\begin{abstract}
Background: Endobronchial navigation is performed in a variety of ways, none of which are meeting all the clinicians' needs required to reach diagnostic success in every patient. We sought to characterize precurved and steerable guiding sheaths (GS) in endobronchial targeting for lung biopsy using cone beam computed tomography (CBCT) based augmented fluoroscopy (AF) image guidance.

Methods: Four precurved GS (Edge ${ }^{\mathrm{TM}} 45,90,180,180 \mathrm{EW}$, Medtronic) and two steerable GS [6.5 F Destino Twist (DT), Oscor; $6 \mathrm{~F}$ Morph, BioCardia] were evaluated alone and in combination with an electromagnetic tracking (EM) guide and biopsy needles in three experimental phases: (I) bench model to assess GS deflection and perform biopsy simulations; (II) ex vivo swine lung comparing 2 steerable and 2 precurved GS; and (III) in vivo male swine lung to deliver a needle ( $\mathrm{n}=2$ swine) or to deliver a fiducial marker (n=2 swine) using 2 steerable GS. Ex vivo and in vivo image guidance was performed with either commercial or prototype AF image guidance software (Philips) based on either prior CT or procedural CBCT. Primary outcomes were GS delivery angle $\left(\theta_{\mathrm{GS}}\right)$ and needle delivery angle $\left(\theta_{\mathrm{N}}\right)$ in bench evaluation and needle delivery error $(\mathrm{mm})$ (mean $\pm \mathrm{se})$ for ex vivo and in vivo studies.

Results: The steerable DT had the largest range of GS delivery angles $\left(\theta_{\mathrm{N}}\right.$ : $\left.0-114^{\circ}\right)$ with either the $21 \mathrm{G}$ or $19 \mathrm{G}$ biopsy needle in the bench model. In ex vivo swine lung, needle delivery errors were $8.7 \pm 0.9 \mathrm{~mm}$ (precurved Edge 90), 5.4 $\pm 1.9 \mathrm{~mm}$ (precurved Edge 180), $4.7 \pm 1.2 \mathrm{~mm}$ (steerable DT), and 5.6 $\pm 2.4 \mathrm{~mm}$ (steerable Morph). In vivo, the needle delivery errors for the steerable GS were $6.0 \pm 1.0 \mathrm{~mm}$ (DT) and $15 \pm 7.0 \mathrm{~mm}$ (Morph). In vivo marker coil delivery was successful for both the steerable DT and morph GS. A case report demonstrated successful needle biopsy with the steerable DT.

Conclusions: Endobronchial needle delivery with AF guidance is feasible without a bronchoscope with steerable GS providing comparable or improved accuracy compared to precurved GS.
\end{abstract}

^ ORCID: 0000-0002-0031-8938. 


\begin{abstract}
Keywords: Lung cancer; cone beam computed tomography (CBCT); augmented fluoroscopy (AF); transbronchial needle aspiration (TBNA); guiding sheaths
\end{abstract}

Submitted Apr 09, 2021. Accepted for publication Jun 25, 2021.

doi: $10.21037 /$ tlcr-21-275

View this article at: https://dx.doi.org/10.21037/tlcr-21-275

\section{Introduction}

Historically, endobronchial lung biopsies have been primarily performed through a bronchoscope, while peripheral lung lesion biopsies have been commonly approached via computed tomography-guided transthoracic needle biopsy (CT-TTNA) (1). However, the higher risk of pneumothorax associated with CT-TTNA compared to transbronchial bronchoscope-guided biopsy has led to the development of imaging and navigation devices to target peripheral lesions $(2,3)$. These devices include virtual bronchoscopy, radial ultrasound, linear endobronchial ultrasound (EBUS), electromagnetic navigation bronchoscopy (ENB), guiding sheaths (GS), and ultrathin bronchoscopes (4-7). Even with these innovations in endobronchial imaging, navigation, and guidance tools over the past decade, the diagnostic yield in real-world settings ranges between $33 \%$ to $73 \%$ (8). Linear EBUS is able to perform simultaneous and real-time needle imaging during device delivery with a favorable valid diagnostic outcome where EBUS can be applied (9-13).

More recent work has evaluated bronchoscope-guided procedures utilizing augmented fluoroscopy (AF) software, CBCT image guidance, or a combination of both. During $\mathrm{AF}$, intraoperative $\mathrm{AF}$ views are extracted from $\mathrm{CT}$ or $\mathrm{CBCT}$ images and superimposed on real-time 2D fluoroscopy images to provide dynamic navigation and treatment planning $(14,15)$. CBCT enables adaptive treatment planning before and during navigation as well as direct assessment of needle delivery since the target and needle can be simultaneously imaged (16-18). Combing CBCT based AF guidance with CBCT assessment of success has improved diagnostic yields for transbronchial needle aspiration $(19,20)$.

CBCT-based AF imaging guidance can also enable bronchoscope-free navigation and biopsy techniques or delivery of local therapeutics that utilize devices capable of overcoming airway size and geometry restrictions that limit bronchoscopy, i.e., endovascular wires, catheters, and guiding sheaths (GS) $(21,22)$. Device selection is also not limited by the dimensions of the bronchoscope working channel. Advancing instruments such as biopsy needles into the working channel of a precurved GS may reduce flexion and steerability of the sheath, compromising navigation performance and delivery accuracy $(23,24)$. Steerable GS allow the operator to deflect the catheter tip to compensate for tip deformation by applying torque and maintaining required needle delivery angles while advancing biopsy instruments into target lesions. Steerable GS combine the attributes of bronchoscope steerability and the precurved GS outer diameters and have shown evidence of improved navigation and steerability in endovascular procedures (18-20).

This study characterized the performance of steerable versus precurved GS compared to precurved GS in a bench model and ex vivo swine lung and evaluated the feasibility of steerable GS in combination with CT and CBCT-based $\mathrm{AF}$ imaging guidance for transbronchial needle delivery and endobronchial fiducial marker delivery in vivo. A healthy swine model provides a cardiopulmonary system comparable to humans to evaluate human factors, identify the optimal device combination, and define and improve the procedural workflow of the device-imaging combination prior to human use (25). The hypothesis was that steerable GS with a deflectable tip could perform accurate transbronchial needle delivery without a bronchoscope and have improved steerability and direction compared to precurved GS, particularly in a steep delivery angle setting to a lung target in vivo.

The aim of this work was to characterize the inherent capabilities and limitations of existing guiding catheters and their performance alone without a bronchoscope. A case report of a successful endobronchial biopsy performed with a steerable GS is presented. We present the following article in accordance with the ARRIVE reporting checklist (available at https://dx.doi.org/10.21037/tlcr-21-275).

\section{Methods}

\section{Preclinical study design}

Precurved endobronchial GS for use with electromagnetic (EM) systems and steerable endovascular GS were evaluated alone and in combination with endobronchial biopsy 


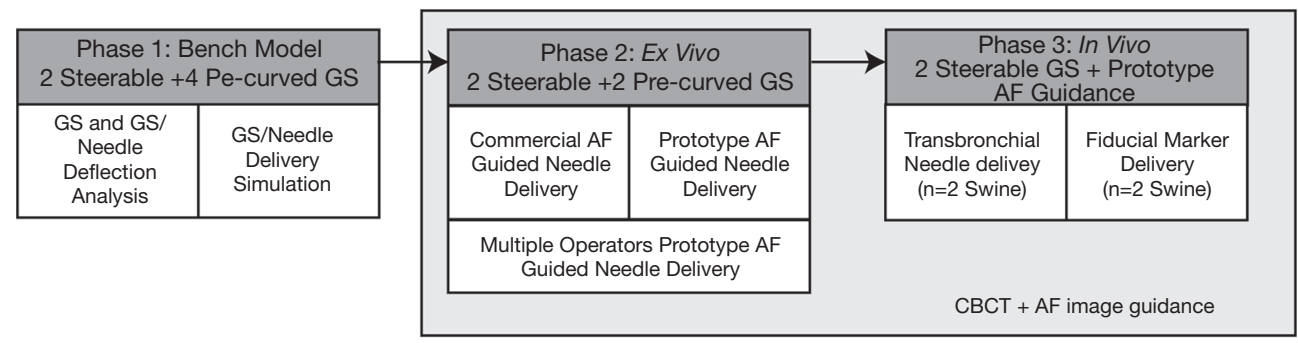

Figure 1 Overview of the experimental study design. Phase 1: All GS were evaluated in a bench model to assess GS deflection, alone and in combination with various biopsy needles to simulate needle deliveries in five biopsy scenarios. Phase 2: Two precurved and two steerable GS were evaluated in an ex vivo lung model using CBCT and AF image guidance to assess needle delivery error across multiple operators. Commercial AF software and prototype AF software with additional capabilities were used. Phase 3: The steerable GS were evaluated in an in vivo swine model using CBCT and prototype AF image guidance to assess the feasibility of endobronchial delivery, including transbronchial needle and fiducial marker delivery. GS, guiding sheath; AF, augmented fluoroscopy; CBCT, cone beam computed tomography.

A
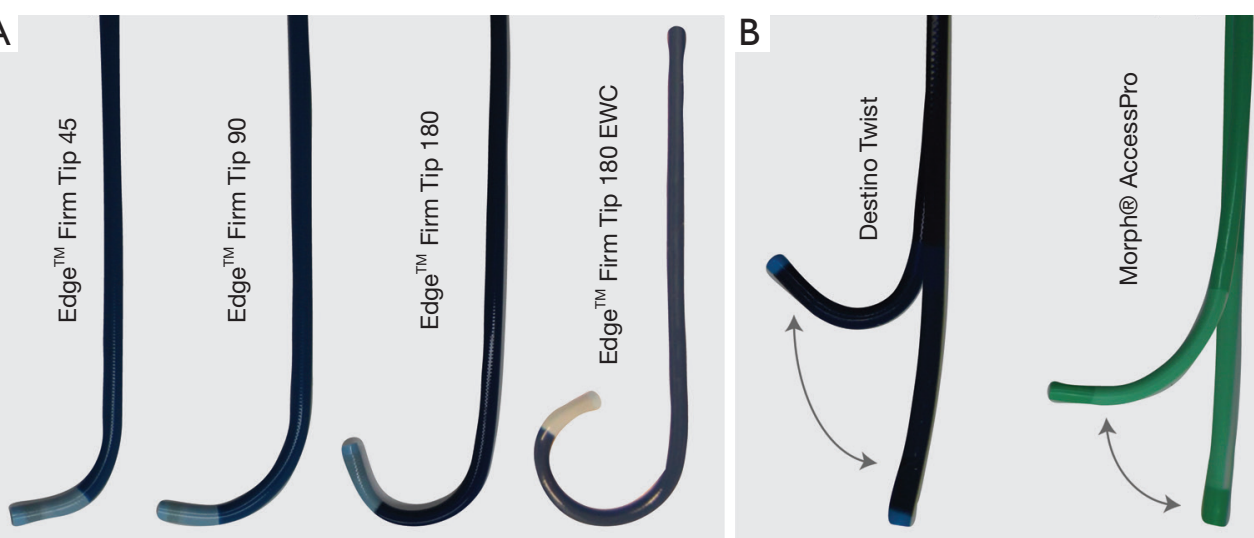

Figure 2 Photographs of the evaluated steerable and precurved GS. (A) Precurved GS (45, 90, 180, and 180 EWC (Medtronic). (B) Steerable GS: 6.5 F Destino Twist (Oscor, Inc.) and the 6 F Morph AccessPro (BioCardia) with images of the straight and flexed positions superimposed. EWC, extended working channel; GS, guiding sheath.

needles in three experimental phases: (I) bench model (4 precurved and 2 steerable GS); (II) ex vivo swine lung ( 2 precurved and 2 steerable GS); and (III) in vivo swine lung (2 steerable GS). In each sequential experimental phase, a subset of GS to test was selected based on the previous phase results (Figure 1).

\section{Devices}

Four commercially available endobronchial GS with precurved tips were studied: the Edge ${ }^{\mathrm{TM}}$ Firm Tip $45^{\circ}, 90^{\circ}$, and $180^{\circ}$ and the Edge EWC Firm Tip $180^{\circ}$ (Medtronic, Minneapolis, MN, USA) (Figure 2). In the ex vivo lung studies, a single-use flexible bronchoscope (aScope 4
Broncho Regular 5.0/2.2 ${ }^{\mathrm{TM}}$, Ambu ${ }^{\circledR}$, Columbia, MD) was selected for bronchoscopy procedures.

The steerable GS were selected based on 4 criteria: (I) minimum of 1 degree of freedom in GS tip steering, (II) a minimum working channel diameter $>2.0 \mathrm{~mm}$ to accommodate most biopsy needles, (III) $<3 \mathrm{~mm}$ outer diameter, comparable to that of an ultrathin bronchoscope, (IV) overall working length $>80 \mathrm{~cm}$, and (V) commercially available. Two endovascular steerable GS were selected: the 6.5 F Destino Twist (DT) (2.2 mm inner working channel, $90 \mathrm{~cm}$ working length, $108 \mathrm{~cm}$ total length, $9 \mathrm{~mm}$ curve bend tip, Oscor Inc, Palm Harbor, FL, USA) and the $6 \mathrm{~F}$ Morph ${ }^{\circledR}$ AccessPro $(2.2 \mathrm{~mm}$ inner working channel, $90 \mathrm{~cm}$ working length, $106 \mathrm{~cm}$ total length, Biocardia, San Carlos, 

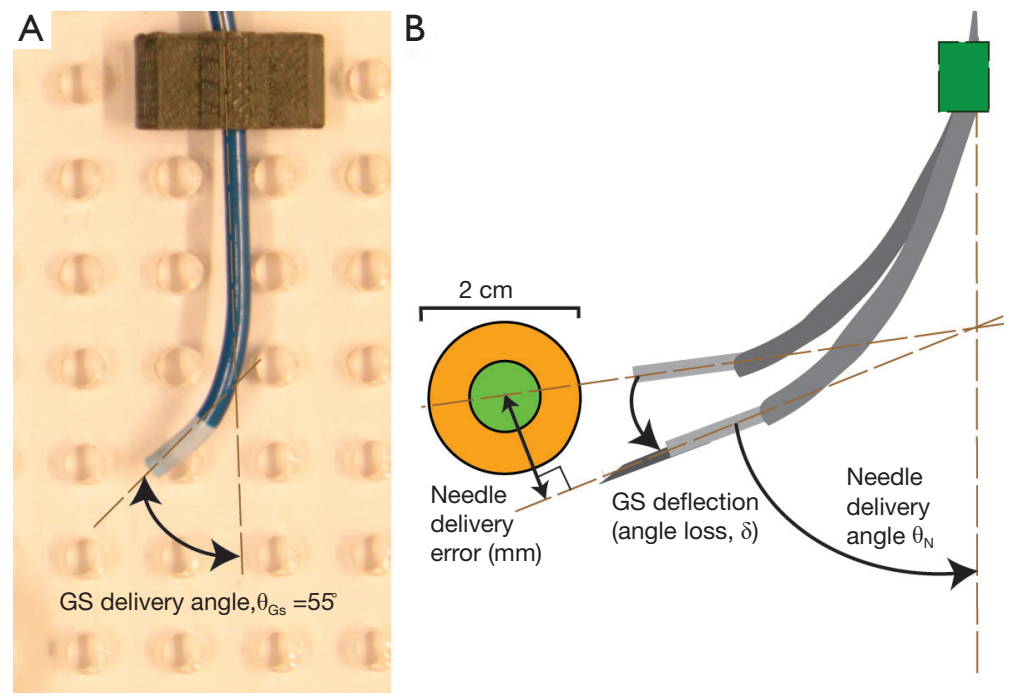

Figure 3 Measurement of baseline GS delivery angle and needle-induced deflection and needle delivery error in a bench model. (A) The shaft of the GS was fixed to the model with a clip $3 \mathrm{~cm}$ from the distal end. The GS delivery angle $\left(\theta_{\mathrm{GS}}\right)$ was defined as the angle between the line extending from the straight portion of the GS shaft and the line extending from the distal tip of the GS without an instrument in the working channel (Edge ${ }^{\mathrm{TM}}$ Firm Tip $90^{\circ}$ shown). (B) Needle delivery angle $\left(\theta_{\mathrm{N}}\right)$ was defined similarly to the GS delivery angle but was measured with a needle in the working channel. The instrument-induced angle loss $(\delta)$ was the difference between the GS delivery angle and the needle delivery angle. The needle delivery error was defined as the shortest distance between the target center and the line extended along the needle direction (B). GS, guiding sheath.

\section{CA, USA) (Figure 2).}

Endobronchial working channel instruments that were evaluated with each GS included the EM locatable guide with a blunt tip (Edge ${ }^{\mathrm{TM}}$ Locatable Guide); $21 \mathrm{G}^{*} 8 \mathrm{~mm}$ aspiration needle; $19 \mathrm{G}^{*} 8 \mathrm{~mm}$ aspiration needle (superDimension, Medtronic, Minneapolis, MN, USA) and an EM trackable Transbronchial access tool (TBAT) (superDimension, Medtronic, Minneapolis, MN, USA).

A $4 \mathrm{Fr}$ multipurpose guide catheter (MPA2, $125 \mathrm{~cm}$, Cordis, Miami Lakes, FL, USA) and guidewire (Glidewire, 0.035 ", $180 \mathrm{~cm}, 3 \mathrm{~cm}$ flexible tip length, angled tip, Terumo Medical, Somerset, NJ, USA) were used to support GS endobronchial navigation without a bronchoscope in the ex vivo and in vivo needle deliveries. For all endobronchial image-guided navigations the devices were introduced into the endotracheal tube via a $9 \mathrm{Fr}$ introducer sheath inserted into a T-shaped single-use valve connected to the endotracheal tube.

\section{Phase 1: bench model}

To simulate the GS needle delivery angles and measure GS deflection, a bench model was developed (see Appendix 1 for bench model description).

\section{Deflection analysis}

To measure the GS delivery angle $\left(\theta_{\mathrm{GS}}\right)$ without an instrument in the working channel, each GS was attached on the grid of the simulation model in the natural angle for precurved GS, and maximally deflected configuration for steerable GS (Figure 3A). An instrument was then advanced through the working channel and out of the GS to measure needle delivery angle $\left(\theta_{\mathrm{N}}\right)$ (Figure $3 B$ ). The baseline GS delivery angle $\left(\theta_{\mathrm{GS}}\right), \mathrm{EM}$ delivery angle $\left(\theta_{\mathrm{EM}}\right)$ with the EM locatable guide, and the needle delivery angle $\left(\theta_{N}\right)$ were evaluated for all possible GS and needle combinations. The instrument-induced GS angle loss $(\delta)$ was defined as the difference between the EM delivery angle $\left(\theta_{\mathrm{EM}}\right)$ and the needle delivery angle $\left(\theta_{\mathrm{N}}\right)$. For the steerable GS, the instrument-induced GS angle loss ( $\delta$ ) was defined as the difference between the baseline maximum GS needle delivery angle and the needle delivery angle.

\section{Needle delivery simulation}

All GS with instrument combinations were evaluated in five needle delivery scenarios that were created on the bench 
model with simulated targets in the lower, middle, and upper lung at $15^{\circ}, 30^{\circ}, 50^{\circ}, 55^{\circ}$, and $65^{\circ}$ approach angles.

For the precurved GS, the GS with the EM locatable guide was pointed towards the center of the target. The EM guide was withdrawn, and the biopsy needle was inserted and advanced out of the GS working channel towards the target, followed by measurement of the needle delivery error (Figure 3B). For the steerable GS, the GS tip was adjusted with the steering handle such that it was pointed towards the center of the target. The biopsy needle was introduced into the working channel and advanced out of the GS towards the target without additional manipulation or rotating the GS steering handle, followed by a measurement of the needle delivery error (no steering). A second attempt with the steerable GS was included in which the operator was allowed to use the GS steering handle to compensate for the needle-induced deflection while advancing the needle out of the working channel and reaching the target center (steering).

The needle delivery error was defined as the absolute distance between the center of the target and the extended line from the distal tip of the GS (Figure 3B). Needle delivery errors were stratified into three categories based on the American Cancer Society non-small cell lung cancer TNM $8^{\text {th }}$ edition defining needle delivery success (26): errors less than $5 \mathrm{~mm}$ were classified as "success to target a $1 \mathrm{~cm}$ tumor" (NSCLC stage IA1); errors between 5 and $10 \mathrm{~mm}$ were classified as "success to target a 2 -cm tumor" (stage IA2); and errors larger than $10 \mathrm{~mm}$ were classified as a "failure to target both the 1 and $2 \mathrm{~cm}$ tumors" (stage IA3).

\section{Ex vivo and in vivo study procedures Image guidance}

All ex vivo (phase 2) and in vivo (phase 3 ) endobronchial navigations and needle deliveries were performed in an interventional radiology laboratory under fluoroscopic and CBCT image guidance (Allura Xper FD20 X-ray System, Philips). The planning CT was acquired either with CBCT or on a multidetector CT (Philips Brilliance MX8000 IDT 16-section Detector CT; Philips, Andover, MA, USA). Fluoroscopic images were acquired at the lowest dose setting at 15 frames/sec. CBCT scans were acquired using the "chest roll" CBCT protocol $(120 \mathrm{kV}$, $60 \mathrm{fps} / \mathrm{sec}, 8 \mathrm{sec}$ acquisition time, 384 images, rotation from +90 to -90 degrees). AF images were generated by extracting anatomic and planning features based on a preoperative $\mathrm{CT}$, a planning CBCT, or a confirmatory CBCT with AF software, and then superimposed on real- time fluoroscopy. The geometric correspondence between real-time fluoroscopy and the superimposed $3 \mathrm{D} \mathrm{AF}$ images, or CT-to-body convergence, is maintained throughout the procedure, independent of $\mathrm{C}$-arm rotation, table position, and magnification settings.

Image guidance was performed with either a commercially available AF software (LungSuite, Philips, Best, NL) intended for needle guidance procedures or a prototype $\mathrm{AF}$ software (Philips, Best, NL) developed for endobronchial guided procedures (22). While both AF software facilitated tumor segmentation, the prototype software had features that enhanced AF workflow, including CBCT airway segmentation, navigation pathway trajectory analysis, and CT-CBCT registration and needle planning. The prototype AF software workflow for endobronchial navigation and needle delivery to a lung target consisted of four procedural steps (I) AF planning, (II) navigation of endobronchial devices, (III) assessment of the catheter or needle position, and (IV) adjustment of needle position (Figure 4).

\section{Endobronchial navigation and needle delivery}

The guidewire and catheter were inserted into the GS and were introduced into the airway through the endotracheal tube. Using the AF guidance, the devices were navigated along the airway leading to the target, rotating the $\mathrm{C}$-arm as needed to optimize visualization and navigation at airway branch points. Once in the desired airway, the C-arm was rotated to obtain a view perpendicular to the target airway. The guidewire was navigated into the airway, and the correct position was confirmed with AF using multiple $\mathrm{C}$-arm angles. Using the same techniques of multi-angle imaging, the tip of the GS was advanced to the preplanned airway exit point for the needle delivery. The guidewire and catheter were then exchanged with a $21 \mathrm{G}$ biopsy needle. Multiple $\mathrm{C}$-arm angles and $\mathrm{AF}$ views were acquired to confirm the needle and GS alignment with the planned needle trajectory prior to needle deployment. The needle was advanced to the virtual target, and a confirmatory CBCT was acquired to assess needle delivery error and to update the superimposed $\mathrm{AF}$ images.

\section{Phase 2: ex vivo lung model}

GS navigation and needle deliveries were conducted in an inflatable plasticized swine lung (see Appendix 1 for $e x$ vivo lung model description). A single operator with $\mathrm{AF}$ and CBCT image guidance experience performed all GS navigation and needle deliveries using four different GS (2 precurved: $\mathrm{Edge}^{\mathrm{TM}} 45$ and Edge ${ }^{\mathrm{TM}}$ 90; and 2 steerable: 
1. Planning

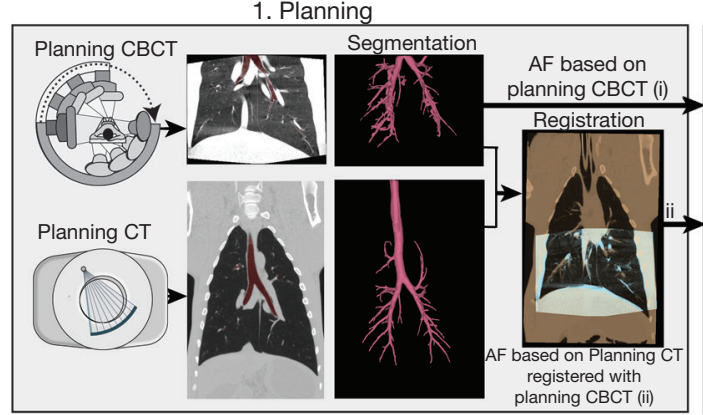

2. Navigation and Guidance

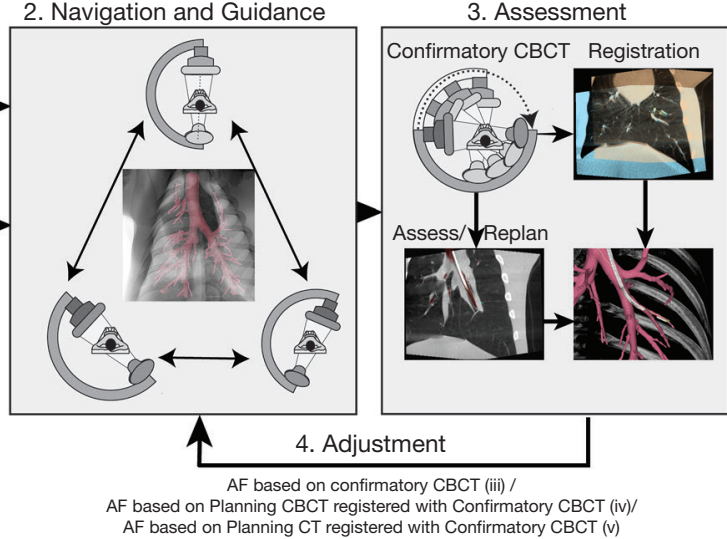

Figure 4 CT, CBCT, and AF image-guided navigation workflow with the prototype software. 1. Planning: a planning CBCT scan is acquired, or a preoperative planning CT is acquired and imported into the system. Automatic bone and airway segmentation is followed by manual segmentation of targets and automatic generation of a centerline to enhance visualization of the navigation pathway from the trachea to the target. The registered segmented airway, targets, and navigation pathways are superimposed onto real-time fluoroscopic imaging (i). In this normal animal shown, there were no lung lesions; the target is virtual. If AF planning were performed on a preoperative CT scan, image registration permits superimposition of the preoperative planning and CT on intraoperative CBCT and real-time fluoroscopy (ii). 2. Navigation and guidance: Fluoroscopic imaging at multiple C-arm angles is used to guide the bronchoscope, GS, and needle delivery. 3 . Assessment: A confirmatory CBCT is acquired to assess needle delivery accuracy or to update the AF treatment planning. 4. Adjustment: Updated AF planning based on confirmatory CBCT can replace the original AF to guide repositioning of devices if required either by direct overlay of the confirmatory CBCT (iii), or by AF based on planning registered with confirmatory CBCT (iv) or AF based on planning CT registered with confirmatory CBCT (v). GS, guiding sheath; AF, augmented fluoroscopy; CBCT, cone beam computed tomography.

Morph and DT), with either the $21 \mathrm{G}$ or the TBAT in the GS working channel. Both the commercial and prototype AF image guidance software were used. In addition, four operators (1 pulmonologist, 1 interventional pulmonologist, 1 interventional radiologist and 1 interventional experimentalist) each subsequently performed 1 to 2 navigation and delivery tasks with the steerable DT GS combined with the TBAT needle using the prototype AF image guidance software. After the confirmatory CBCT for the first attempt at delivery, operators were allowed to adjust the needle position, if necessary. Repositioning used updated AF image views derived from the confirmatory CBCT prior to advancing the needle into the target. The primary endpoint was the needle delivery error ( $\mathrm{mm}$ ) defined as the shortest distance from the edge of the bead target and the line extended along the needle direction and was assessed on the 3D CBCT acquisition using the commercial AF software.

\section{Phase 3: In vivo lung study}

Experiments were performed under an animal study protocol (DRD 17-01) approved by the institutional Animal Care and Use Committee (ACUC) in compliance with the Public Health Service (PHS) Policy on Humane Care and Use of Laboratory Animals (policy) and was reported following the ARRIVE guidelines (See Appendix 1 for swine management and anesthesia details). Four healthy castrated male Yorkshire swine were studied $(28 \pm 15$ weeks: $55.5 \pm 15 \mathrm{~kg}$ ) (Oak Hill Genetics, Ewing, IL), two swine to evaluate transbronchial needle delivery and two swine to evaluate fiducial marker delivery as defined before the study.

No blinding, control group, randomization, or sample size calculations was conducted, as the work was performed as a pilot feasibility study. The number of lung target deliveries per animal study was based on predicted delivery time to each lung target not exceeding the total approved anesthesia time for the animal study protocol (8 hours per animal).

A CT scan with a breath hold at end-expiration was acquired on each subject. The subject was then placed on the fluoroscopy CBCT table, and an end-expiration CBCT scan was acquired. The reconstructed CT was registered with the CBCT to generate CT-based AF (Figure 4), and endobronchial procedures with steerable GS were conducted. 


\section{Transbronchial needle delivery ( $\mathrm{n}=2$ swine)}

Eight virtual peripheral landmarks (four in each swine) were defined on the planning CT, $2-3 \mathrm{~cm}$ lateral to a $3^{\text {rd }}$ generation bifurcation to serve as the target for each needle delivery. Four needle deliveries were planned for each GS. Swine \#1 was assigned to evaluate the DT GS, and swine \#2 was assigned to evaluate the feasibility of the Morph GS. As this was a feasibility study, no blinding or randomization was performed. The operator was allowed to reposition the needle up to three times for each target, with $\mathrm{CBCT}$ conducted for confirmation and $\mathrm{AF}$ adjustment pre-and post- needle delivery final assessment. In the first two navigation and needle delivery tasks in swine 1 with the DT, the initial needle delivery was guided by CT-based $\mathrm{AF}$ views. $\mathrm{CBCT}$ was then used for position confirmation and further AF image guidance. In the subsequent six tasks ( 2 tasks in swine 1 with the DT and 4 tasks in swine 2 with the Morph), initial navigation was performed using CTbased $\mathrm{AF}$ views, but a confirmatory $\mathrm{CBCT}$ was acquired and used for AF guidance during the first attempt at needle delivery. CBCT was then again used for needle position confirmation and further guidance. A new pre-curved GS was substituted when a significant change in the resting curve was noted on the bench. A new steerable GS was used for each animal and assessed prior to each target delivery for acceptable performance.

The primary endpoint was the needle delivery error $(\mathrm{mm})$ measured on the final confirmatory CBCT. Secondary endpoints were GS navigation success and the learning curve. Evaluation of the learning curve effect for in vivo needle delivery was based on changes in workflow, procedural and fluoroscopy time, and the number of confirmatory CBCT between the first two and the subsequent six needle deliveries. The needle delivery error was defined as the distance from the center of the virtual target to the centerline of the needle. This was assessed on the confirmatory CBCT using the prototype AF software by growing a margin volume around the target lesion until the margin overlapped with the delivered needle. The needle delivery error was the sum of the radius of the target and the thickness of the added margin.

\section{Fiducial marker delivery ( $\mathrm{n}=\mathbf{2}$ swine)}

Both steerable GS were evaluated to deliver a fiducial marker in a bronchial segment using CBCT-based AF (swine \#3, 4 fiducial markers with DT, and swine \#4, 3 fiducial markers with the Morph). Fourth to $5^{\text {th }}$ generation airway segments were marked with virtual landmarks on the planning CBCT to identify the target sites for delivery of fiducial markers. A steerable GS was navigated under AF image guidance over a catheter and guidewire to a major airway branch point along the navigation pathway. The GS tip position was confirmed using multiple $\mathrm{AF}$ views, and the catheter and guidewire were then advanced to the target airway segment. The fiducial marker $(2 \mathrm{~cm}$, stainless steel coil, Cook Medical, Bloomington, IL, USA) was advanced through the catheter and released at the target location. A confirmatory CBCT was acquired to confirm marker delivery location. The primary endpoint was the fiducial marker delivery success, defined as a full delivery of the coil out of the sheath into the target branch and measured on the final confirmatory CBCT.

\section{Statistical analysis}

GS navigation success was defined as navigating the GS to the target airway and positioning the GS needle tip with the planned needle trajectory to obtain a safe needle delivery. If GS navigation was not successful, needle delivery was not performed, and the needle delivery error was excluded from the overall needle delivery error calculation. Needle delivery errors were expressed as mean \pm se.

Post- needle delivery CBCT images were evaluated by a radiologist for needle delivery-related complications, including pneumothorax, pulmonary hemorrhage as represented by new consolidation or ground-glass opacity near the needle delivery site. The severity of consolidation was graded on a four-point scale: $0=$ no consolidation or $<1 \mathrm{~cm} ; 1=$ consolidation or ground-glass opacity $1-2 \mathrm{~cm}$ in width; $2=$ consolidation or ground-glass opacity $>2 \mathrm{~cm}$ in width but sublobar and; $3=$ as lobar consolidation or ground-glass opacity or greater (27).

A standard $t$-test was performed to assess needle delivery error. Statistical analysis was performed using a statistical software program R (R Core Team (2020). R: A language and environment for statistical computing. R Foundation for Statistical Computing, Vienna, Austria. https://www. R-project.org/).

\section{Results}

\section{Phase 1: bench model}

\section{Deflection analysis}

Tables 1,2 summarize the baseline GS delivery angle and needle-induced angle loss for each GS/needle combination, 
Table 1 Precurved GS delivery angle before and after introducing the EM guide, 21G, 19G, or TBAT needle in the working channel. Percent angle loss $(\delta)$ is expressed as the percent change in delivery angle $(\theta)$ between GS with EM guide in the working channel ( $\left.\theta_{\mathrm{EM}}\right)$ and the needle delivery angle $\left(\theta_{\mathrm{N}}\right)$ with a $21 \mathrm{G}, 19 \mathrm{G}$, or TBAT needle in the working channel

\begin{tabular}{|c|c|c|c|c|c|}
\hline Precurved GS & $\frac{\text { GS delivery angle }\left(\theta_{\mathrm{GS}}\right)}{\text { Baseline }}$ & $\begin{array}{c}\text { GS/EM delivery angle }\left(\theta_{\mathrm{EM}}\right) \\
+ \text { EM guide }\end{array}$ & \multicolumn{3}{|c|}{ GS/needle delivery angle, $\theta_{\mathrm{N}}\left(\%\right.$ angle loss from $\left.\theta_{\mathrm{EM}}\right)$} \\
\hline Edge 45 & $45^{\circ}$ & $40^{\circ}$ & $39^{\circ}(-2.5 \%)$ & $37^{\circ}(-7.5 \%)$ & $12^{\circ}(-70 \%)$ \\
\hline Edge 90 & $90^{\circ}$ & $57^{\circ}$ & $45^{\circ}(-21 \%)$ & $40^{\circ}(-30 \%)$ & $21^{\circ}(-63 \%)$ \\
\hline Edge 180 & $180^{\circ}$ & $96^{\circ}$ & $54^{\circ}(-44 \%)$ & $50^{\circ}(-48 \%)$ & $25^{\circ}(-74 \%)$ \\
\hline
\end{tabular}

N, needle; GS, guiding sheath; EM, electromagnetic tracking; TBAT, transbronchial access tool; EWC, extended working channel.

Table 2 Steerable GS maximum delivery angle before and after introducing a 21G, 19G, or TBAT needle in the working channel

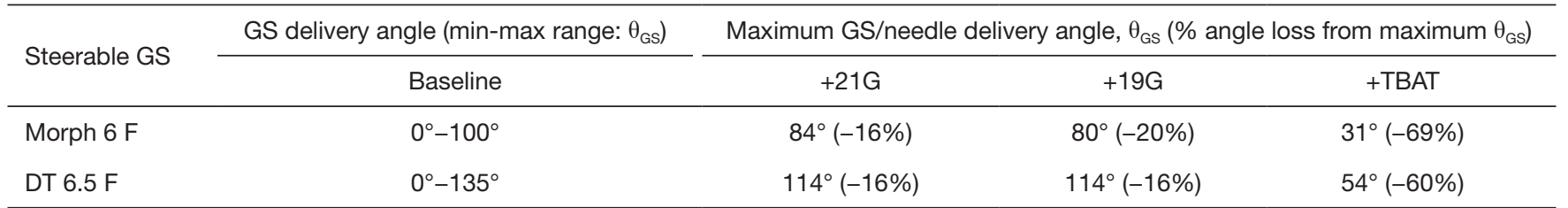

Percent angle loss is expressed as the percent change between the maximum GS delivery angle $\left(\theta_{\text {GS }}\right)$ and GS/needle delivery angle $(\mathrm{N})$ with a 21G,19G, or TBAT needle in the working channel. DT, Destino Twist; TBAT, transbronchial access tool; GS, guiding sheath.

as well as the delivery angle for the EM guide for the precurved GS. For precurved GS, needle-induced angle loss ranged from $2.5 \%$ for the Edge $45 / 21 \mathrm{G}$ combination to $74 \%$ for the Edge 180/TBAT combination (Table 1). Across all GS evaluated, the steerable DT was capable of the largest needle delivery angle of $114^{\circ}$ with either the $21 \mathrm{G}$ or $19 \mathrm{G}$ biopsy needle in the working channel (Table 2). The maximum delivery angle without an instrument in the steerable GS working channel was $135^{\circ}$ for the DT and $100^{\circ}$ for the Morph. The $21 \mathrm{G}$ and $19 \mathrm{G}$ biopsy needles induced a $16-20 \%$ loss in the Morph and a 16\% loss in the DT in the maximum delivery angle. The TBAT-induced a $69 \%$ angle loss in the Morph maximum delivery angle to $31^{\circ}$ and a $60 \%$ loss in DT maximum needle delivery angle to $54^{\circ}$.

\section{Needle delivery simulation}

Representative needle delivery simulations with precurved GS (Edge 90) and steerable GS (DT) are demonstrated on the bench model in Figure 5 .

The precurved GS (Edge 45, 90, 180, and 180 EWC), when combined with an EM guide, successfully aimed the catheter tip toward a 2 -cm target (error $<10 \mathrm{~mm}$ ) in $90 \%$ $(18 / 20)$ of all needle delivery scenarios. However, the target success rate decreased to $45 \%(9 / 20), 40 \%(8 / 20)$, and $20 \%$ (4/20) when the EM guide was replaced with a $21 \mathrm{G}, 19 \mathrm{G}$, or TBAT needle, respectively. The mean needle delivery error for the precurved GS using either the $21 \mathrm{G}$ or $19 \mathrm{G}$ needles was $21.8 \pm 11.1 \mathrm{~mm}$ with the Edge $45,17.9 \pm 7 \mathrm{~mm}$ with the Edge 90 (Figure 5B), 26 $\pm 14.3 \mathrm{~mm}$ with the Edge 180 , and $15.4 \pm 13 \mathrm{~mm}$ with the Edge 180 EWC.

The mean DT GS needle error for all delivery combinations was greater without steering than with steering $(16.2 \pm 8.2$ vs. $5.4 \pm 7.6 \mathrm{~mm}, \mathrm{P}=0.001)$. Similarly, the Morph GS mean needle delivery error was greater without steering than steering $(16.2 \pm 8.2$ vs. $8.3 \pm 12 \mathrm{~mm}, \mathrm{P}=0.003)$.

At $15^{\circ}$ and $30^{\circ}$ approach angles in the lower and middle lung simulation, precurved GS successfully reached a $2-\mathrm{cm}$ target (error $<10 \mathrm{~mm}$ ) in $83 \%(20 / 24)$, and a $1 \mathrm{~cm}$ target (error $<5 \mathrm{~mm})$ in $21 \%(5 / 24)$ of the scenarios. Steerable GS (Morph and DT), without steering, successfully reached a 2 -cm target $75 \%(9 / 12)$ and $1 \mathrm{~cm}$ target in $8 \%(1 / 12)$ of the scenarios. However, with steering, success reaching a $1 \mathrm{~cm}$ target was $100 \%(12 / 12)$.

At $55^{\circ}$ and $65^{\circ}$ approach angles in the upper lung simulation, all precurved GS and steerable GS without steering failed to reach a $2-\mathrm{cm}$ target for all device combinations in all scenarios. The DT GS with steering successfully reached a $1 \mathrm{~cm}$ target in $83 \%(5 / 6)$ of the scenarios. The single delivery failure occurred with the TBAT in the biopsy scenario with the steepest approach angle. 

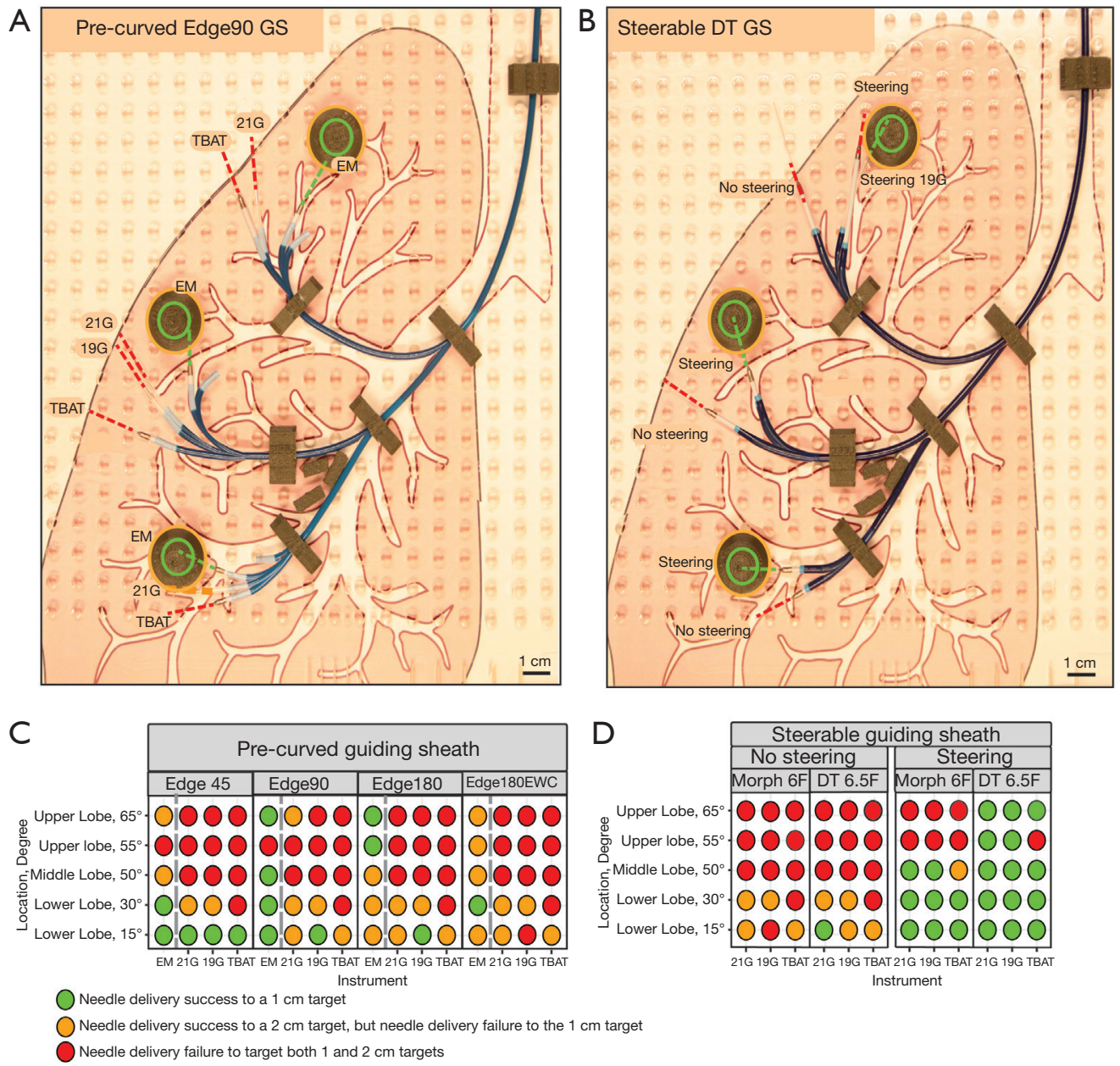

Figure 5 Needle delivery success for each GS/instrument combination. Needle delivery scenarios simulating lower, middle and upper lung targets at $15^{\circ}, 30^{\circ}, 50^{\circ}, 55^{\circ}$, and $65^{\circ}$ approach angles, of which $30^{\circ}, 50^{\circ}$, and $55^{\circ}$ are shown in $\mathrm{A}$ and $\mathrm{B}$, were evaluated on the bench model. Representative images of three GS delivery scenarios are shown in composite photographs for a precurved Edge 90 (A) and steerable DT GS (B): (A) the precurved Edge 90 GS combined with an EM guide demonstrated successful positioning with the tip aimed toward a 1cm target ( $<5 \mathrm{~mm}$ error). However, exchanging the EM guide with a 21G, 19G, or TBAT needle demonstrated failure to deliver to a 2-cm target ( $>10 \mathrm{~mm}$ error). (B) Steerable DT GS combined with a TBAT needle, without steering failed to deliver to a 2-cm target ( $>10 \mathrm{~mm}$ error). However, with DT GS steering TBAT demonstrated delivery success to the lower and middle $1 \mathrm{~cm}$ targets $(<5 \mathrm{~mm}$ error), and with a $21 \mathrm{G}$ or 19G similar target success ( $<5 \mathrm{~mm}$ error) including upper targets. Needle delivery error is categorized for all precurved GS/instrument combinations (C), and all steerable GS/instrument combinations (D): green: delivery within $1 \mathrm{~cm}$ target $(<5 \mathrm{~mm}$ error); orange: delivery within $2 \mathrm{~cm}$ target (5-10 $\mathrm{mm}$ error); red: delivery outside $2 \mathrm{~cm}$ target ( $>10 \mathrm{~mm}$ error). EM, electromagnetic guide; TBAT, transbronchial access tool; GS, guiding sheath; DT, Destino twist; EWC, extended working channel.

\section{Phase 2: ex vivo lung model}

All targets were successfully delivered in the ex vivo swine lung, imaged with CBCT, segmented, and visualized on intraoperative real-time AF views using both the commercial and prototype AF software. Figure 6 illustrates ex vivo AF image-guided navigation of GS to the targets and the needle delivery errors for two precurved and two steerable GS.

For the single operator, no difference in needle delivery error was noted between the precurved and steerable GS while using the commercial AF image guidance application: error was $4.0 \pm 1.8 \mathrm{~mm}$ for the precurved GS and $4.5 \pm 1.2 \mathrm{~mm}$ 
for the steerable GS $(\mathrm{P}=0.847)$. However, a significant difference was noted between the precurved and steerable GS needle delivery success while using the prototype CBCT-based AF image guidance, where the error was $7.2 \pm 1.4 \mathrm{~mm}$ for the precurved GS and $1.9 \pm 0.6 \mathrm{~mm}$ for the steerable $\mathrm{GS}(\mathrm{P}=0.047)$.

All four operators $(n=4)$ in the multiple operator group successfully navigated and delivered the steerable DT GS combined with a TBAT with an overall accuracy of $2.8 \pm 3.6 \mathrm{~mm}$. Three out of four operators were able to deliver the tip of the TBAT needle to directly touch the surface of the solid target in the lung.

\section{Phase 3: in vivo swine model}

\section{Transbronchial needle delivery}

Navigation and delivery of the DT GS to a virtual target using AF image guidance and 3D CBCT image confirmation is illustrated in Figure 7. GS navigation success was $100 \%$ for the DT and $75 \%$ for the Morph. The mean needle delivery error was $6.0 \pm 0.5 \mathrm{~mm}$ for the DT $(\mathrm{n}=4$, swine \#1) and $15 \pm 4.0 \mathrm{~mm}$ for the Morph ( $\mathrm{n}=3$, swine \#2).

Initial navigation and needle delivery to the target with the DT ( $n=2)$ were performed using CT-based prototype AF image guidance. In both cases, a post-delivery confirmatory CBCT identified pulmonary hemorrhage at the target site: grade $1(17 \mathrm{~mm} \times 12 \mathrm{~mm})$ and grade $2(20 \mathrm{~mm} \times 25 \mathrm{~mm})$. For the two needle deliveries, the overall averaged navigation, delivery, and imaging time (from the start of the navigation of the steerable GS to confirmation of the most accurate needle position with CBCT, including replanning) was $44.5 \mathrm{~min}$ with an average of 12.5 fluoroscopy minutes using 3 or 4 confirmatory CBCT scans.

In subsequent $\mathrm{GS}$ navigation and needle delivery tasks $(\mathrm{n}=2$ DT and $\mathrm{n}=3$ Morph), CBCT-based AF views were incorporated prior to the first needle delivery. In these cases, no hemorrhage or pneumothorax was observed post needle delivery at the virtual target sites. The average procedure time was $18.4 \mathrm{~min}$ with an average fluoroscopy time of 7.4 min using 2 to 3 confirmatory CBCT scans for the DT and Morph.

\section{Fiducial marker delivery}

Fiducial marker delivery to virtual targets was $100 \%$ successful with both the DT (4/4, swine \#3) and Morph GS (3/3, swine \#4). Figure 8 illustrates the delivery of a coil using AF image guidance. No hemorrhage or pneumothorax was observed on the post-delivery CBCT scan.

\section{Clinical case report}

The patient reported here underwent a clinically indicated biopsy and consented to the use of endovascular steerable guide sheaths, which was anticipated based on the biopsy target location. The case is reported under a retrospective review protocol that was approved by the Institutional Review Board and met the criteria for waiver of patient consent. A 57-year-old female with bronchiectasis, Marfan Syndrome, and known Mycobacterium avium complex (MAC) presented with a paramediastinal mass in the right lower lobe. The mass was a strong, PET-positive hypermetabolic lesion (max 8.5 SUV), with additional hypermetabolic activity in parenchymal nodules in both right upper and lower lobes. The decision to perform a transbronchial biopsy using $\mathrm{AF}$ and $\mathrm{CBCT}$ guidance was based on nodule classification as a challenging location near major vessels and at high risk of failure with standard bronchoscopy tools. AF was facilitated by the commercial LungSuite modules XperGuide and EmboGuide (Philips, Best, NL) on the interventional workstation with manual segmentation of the airway and target mass. AF treatment planning was performed on $\mathrm{CT}$, which was imported and registered with preoperative CBCT. Initial navigation was performed with a bronchoscope combined with ENB and CT-based AF image guidance and a precurved catheter, but the needle could not be advanced into the lesion. The procedure was converted to the use of DT GS with CT and CBCT-based $\mathrm{AF}$ image guidance without the use of a bronchoscope, performed by an interventional radiologist with experience in catheter-based, image-guided procedures. Figure 9 illustrates the failed approach in performing an ENB-guided bronchoscopic biopsy with a precurved GS and subsequent conversion (Figure 10) to a steerable GS biopsy approach without a bronchoscope. The DT GS was navigated towards the AF planned airway exit point. The sheath's tip was successfully aligned, matching with the AF planned needle delivery angle and airway exit point. The GS tip position and directional delivery angle was confirmed with multiple projections on fluoroscopy. The GS was able to maintain the needle delivery angle while inserting and advancing the needle out of the sheath. CBCT confirmed that the needle delivery angle was correct, but the needle had not yet entered the target lesion. The needle was advanced while maintaining the needle delivery angle, followed by CBCT to confirm that the biopsy needle was positioned within the target. The procedure was followed by bronchoalveolar lavage (BAL) with a flexible bronchoscope; $150 \mathrm{~mL}$ of saline 

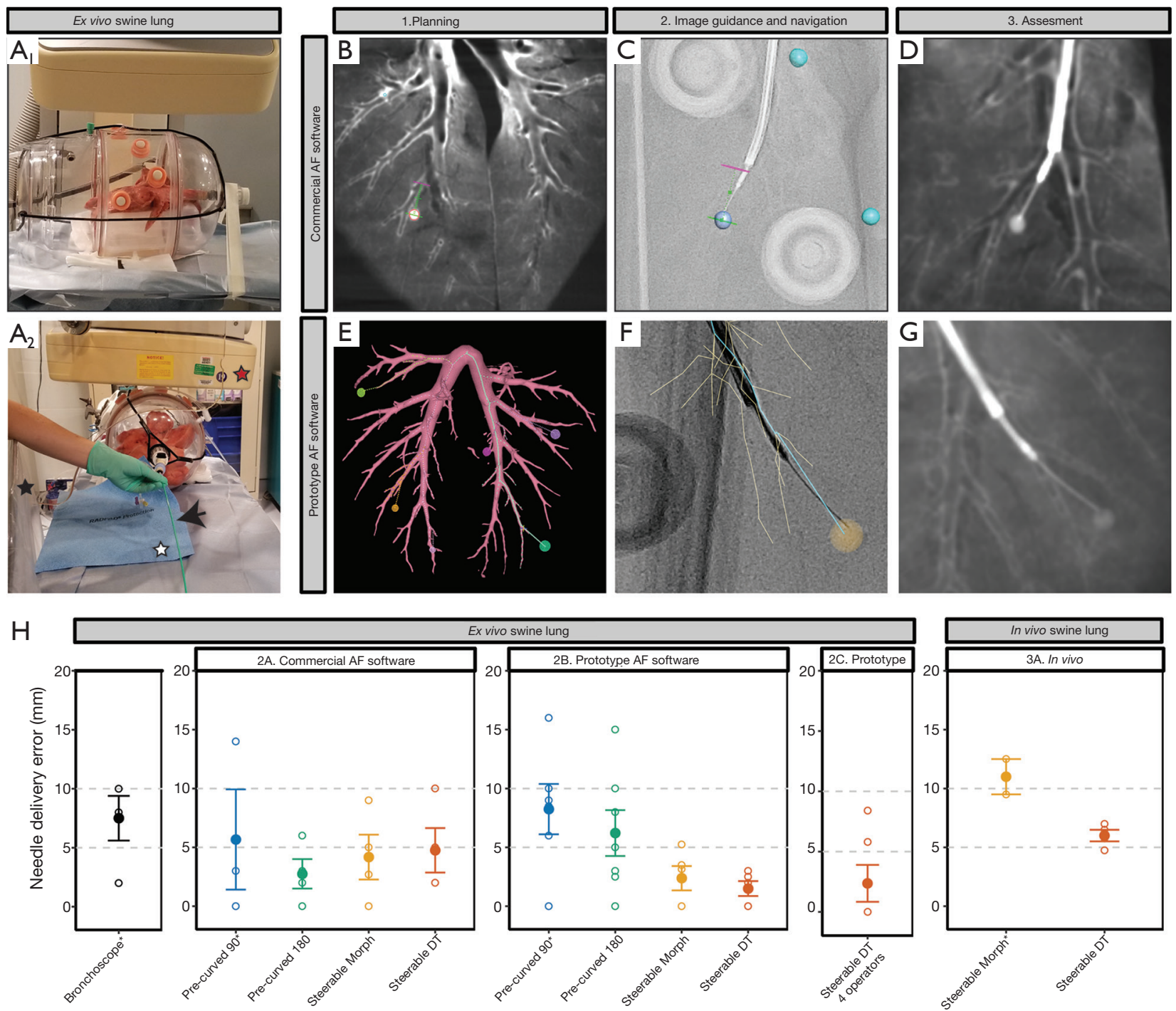

Guiding Sheath

Figure 6 Ex vivo and in vivo image-guided procedural flow, navigation, and needle delivery error. (A1) Side view of the ex vivo swine lung in a vacuum container on the fluoroscopy table. (A2) Front view of the ex vivo swine lung model with a Morph guiding sheath (GS) (arrow) positioned in the bronchial airway with the intention of radiation safety: and a blue radiation-absorbing blanket that should optimally be placed over the subject body, ceiling-mounted lead screen (black star) and detector positioned to minimize air gap and radiation scatter to the operator (red star). (B-G) Use of commercially available augmented fluoroscopy (AF) software (B-D) and prototype augmented fluoroscopy (AF) software (E-G) for planning (B,E), image guidance and navigation (C,F), and assessment of GS navigation and needle delivery (TBAT) to the target $(\mathrm{D}, \mathrm{G})$. Note that the prototype software incorporated airway segmentation and planning. (H) Comparison of precurved and steerable GS needle combination (21G or TBAT) needle delivery error in the ex vivo (gel bead target) and in vivo (peripheral virtual target) swine lung models. Needle delivery error is expressed in mm (mean \pm se). * GS navigation failures that were excluded from the error analysis included: ex vivo bronchoscope $(\mathrm{n}=2)$ and precurved Edge 90 ( $\mathrm{n}=3$ ), and in vivo steerable Morph ( $\mathrm{n}=1$ ). $\mathrm{AF}$, augmented fluoroscopy; DT, Destino twist. 

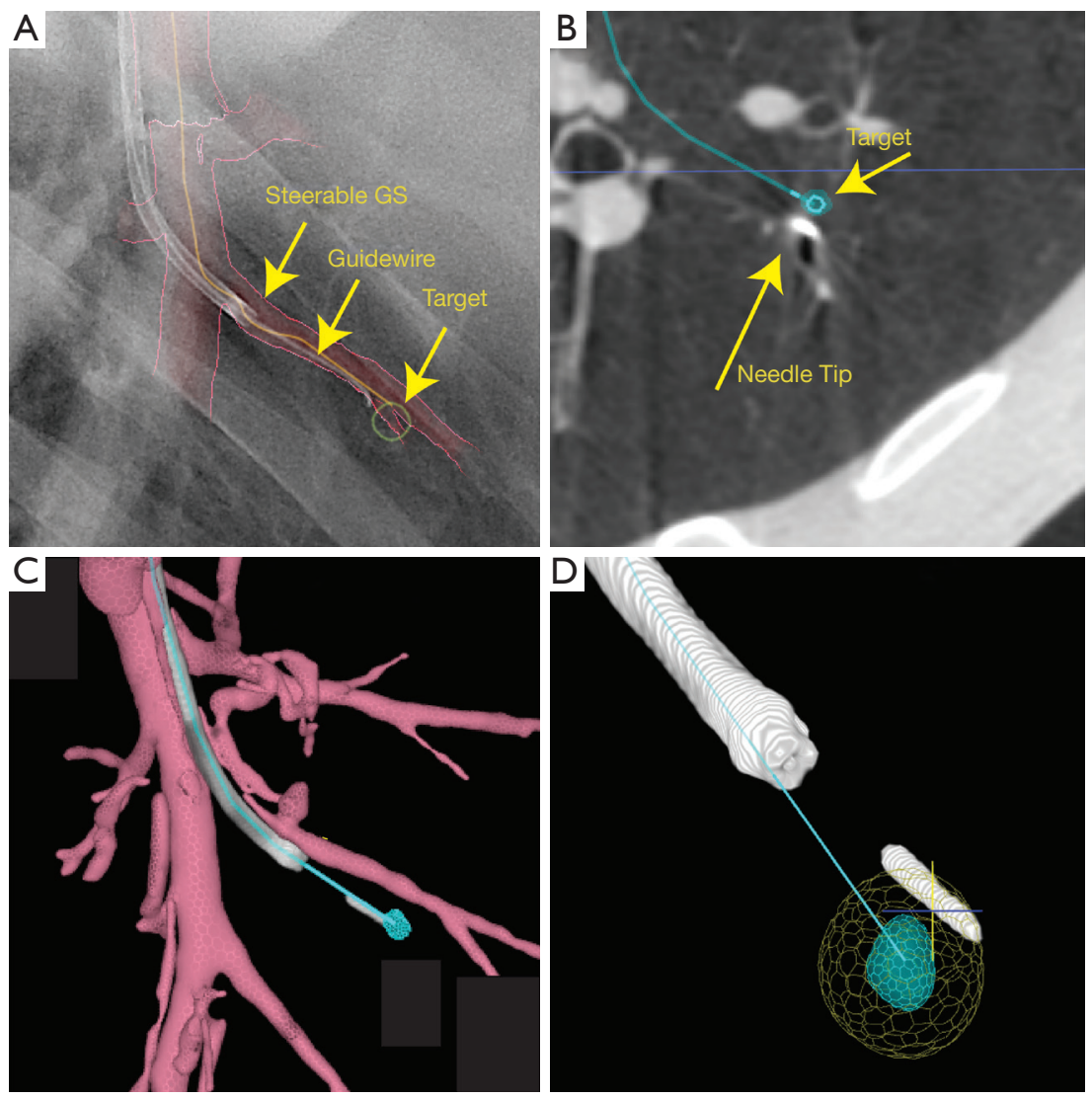

Figure 7 In vivo navigation and delivery of a steerable DT GS and 21G needle combination to a virtual target using prototype AF for image guidance and confirmatory CBCT to assess needle delivery error. (A) AF image guidance showing a steerable DT GS and guidewire with a virtual navigation pathway identified (yellow line) to the target (green circle). (B) Representative axial slice of the confirmatory CBCT showing the post-delivery needle tip adjacent to the virtual target (blue). (C) 3D airway segmentation showing target and navigation pathway based on the planning CBCT and co-registered with confirmatory CBCT after navigation with steerable DT GS and delivery of a $21 \mathrm{G}$ needle to the target (blue). (D) Quantification of needle delivery error (mm) by increasing the margin (yellow) of the virtual target (blue) until the margin volume overlaps with the biopsy needle. GS, guiding sheath.

was instilled, and $40 \mathrm{~mL}$ was aspirated. The total fluoroscopy time was 26.9 minutes. Post-procedure CBCT demonstrated atelectasis in the right lower lobe.

\section{Discussion}

Steerable endovascular GS outperformed precurved endobronchial GS for transbronchial navigation and needle delivery accuracy under CT and CBCT-based AF without the use of a bronchoscope.

The bench studies showed that the precurved endobronchial GS with the EM guide could be successfully directed toward $2 \mathrm{~cm}$ targets, but the GS was deformed once the EM guide was exchanged for a biopsy needle. The
GS deformation altered the needle trajectory leading to failure of the needle to reach the target and errors greater than $10 \mathrm{~mm}$. In contrast, the steerable endovascular GS' (Morph and DT) could maintain optimal needle trajectories and navigate greater approach angles to lung targets with higher success rates and errors less than $5 \mathrm{~mm}$. In the bench model, the DT GS accurately delivered a needle to $1 \mathrm{~cm}$ targets in the upper lobe and at steeper angles with greater accuracy compared to the precurved GS and the Morph. The DT also had a wider range of needle delivery angles (up to 135 degrees) compared to the precurved and Morph GS. These characteristics may allow steerable GS to reach higher-generation airways, navigate at larger GS delivery angles, or deliver biopsy tools capable of obtaining larger 

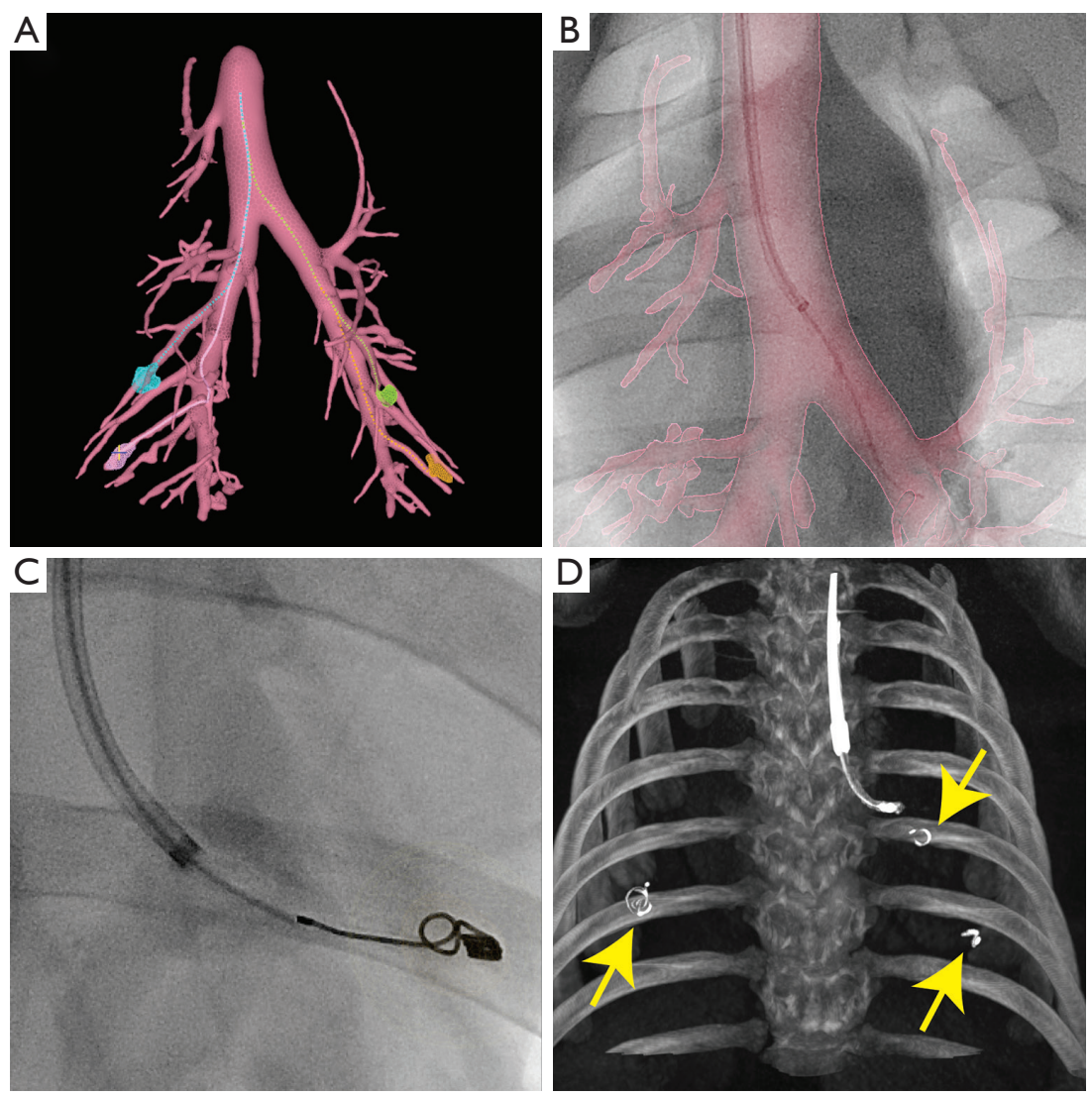

Figure 8 Navigation and delivery of a fiducial marker (coil) with the steerable DT GS using the prototype AF image guidance. (A) AF planning including airway segmentation and navigation pathways to selected endobronchial segment targets for fiducial marker delivery. (B) AF image guidance showing navigation of steerable guiding sheath and the guidewire in the trachea and left mainstem bronchus. (C) Fluoroscopic image during delivery of a fiducial marker (coil) from a 4F catheter delivered via the DT. (D) 3D reconstruction of the confirmatory CBCT, posterior view, including the steerable guiding sheath (GS) with 4F catheter and delivered fiducial markers (yellow arrows). DT, Destino twist; GS, guiding sheath; CBCT, cone beam computed tomography.

tissue samples. These results may be extrapolated to the use of next-generation more flexible needles with reduced stiffness, resulting in reduced GS loss of flexion, thus increasing the maximum needle delivery angle. Although speculative, this may contribute to higher diagnostic yield rates in both adult and pediatric patients $(6,28)$.

The DT demonstrated an overall mean accuracy of 2.4 to $3.4 \mathrm{~mm}$ in the ex vivo study and $6.0 \mathrm{~mm}$ in the in vivo study. These results are comparable to our first report of transbronchial catheter navigation under CBCT-based AF without the use of a bronchoscope with a target accuracy of $4.5 \mathrm{~mm}$ in up to the fifth-generation airways (22).

The outer diameter of steerable GS is slightly smaller than $2.8 \mathrm{~mm}$ ultrathin scopes, while the inner working channels are slightly larger than those of larger bronchoscopes,
2.2 vs. $1.7 \mathrm{~mm}$, respectively. These characteristics may allow steerable GS to reach higher-generation airways and to deliver biopsy tools capable of obtaining larger tissue samples. This may contribute to higher diagnostic success rates in both adult and pediatric patients $(6,28)$. The impact of improved steerability and reduced deflection during needle deployment with steerable GS have been demonstrated in other challenging endovascular navigation tasks or deliveries, including catheter ablation for atrial fibrillation and complex aortic or thoracic aneurysm repair (29-31). Moreover, these procedures have also been performed without intravascular imaging and solely rely on real-time X-ray imaging and CT or CBCT based AF or 3D road mapping image guidance.

The introduction of ENB-guided and precurved GS 

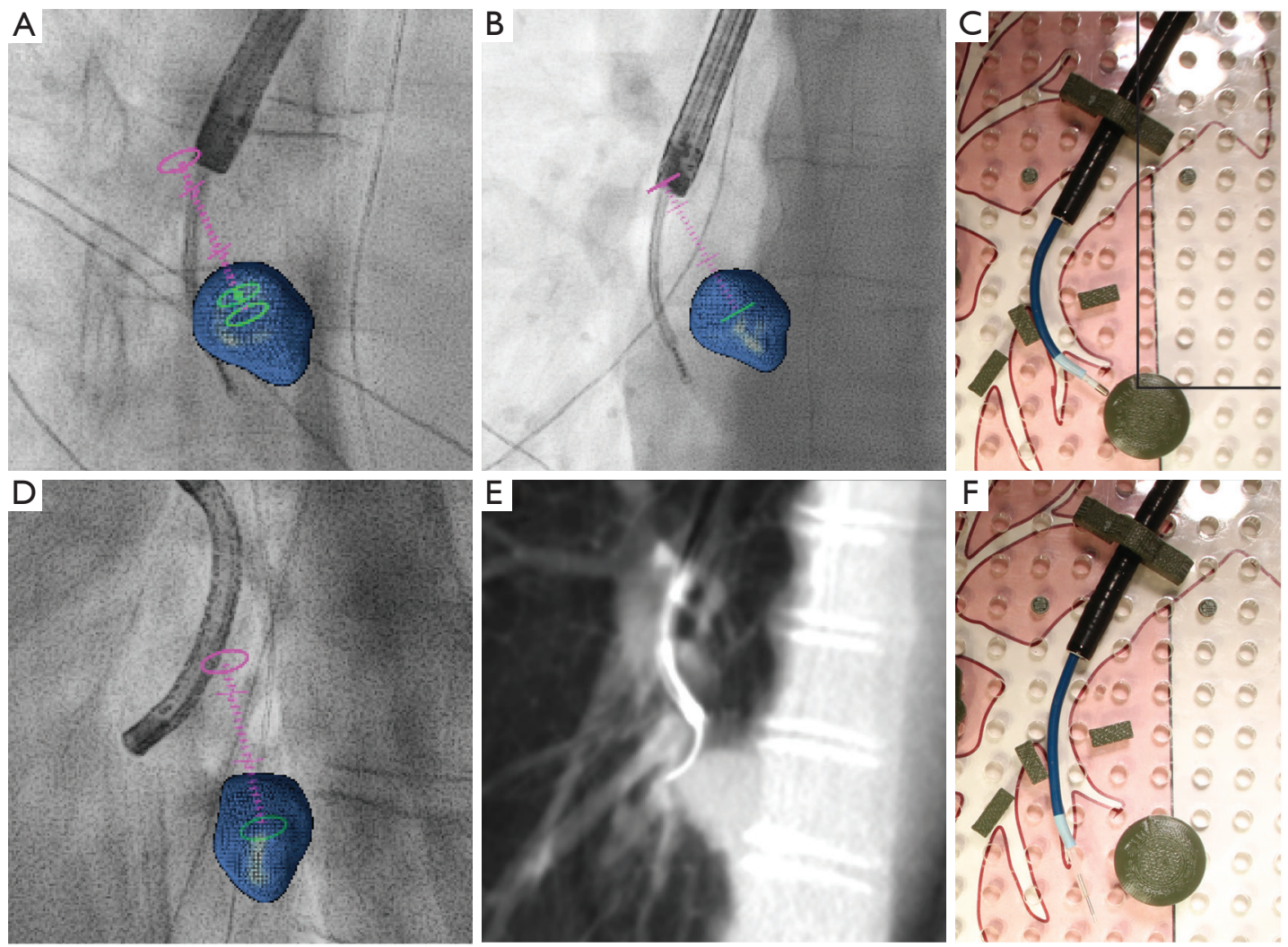

Figure 9 Attempted biopsy of the central lung nodule using a bronchoscope and Edge catheter. (A) The needle is projected over the target nodule in this single fluoroscopy projection. The segmented lesion (blue) and the guidance overlay on the fluoroscopic image are shown, including the planned airway exit point (purple circle), planned needle endpoint (teal circle), and needle trajectory (purple line). (B) A second fluoroscopy projection demonstrated the needle was not in the lesion. (C) Simulation of positioning of the bronchoscope, GS, and EM locatable guide, with the tip pointing towards the lesion. (D,E) A smaller scope is used, in which AF and CBCT showed that the catheter failed to navigate to the lesion. (F) Simulation of the bronchoscope, with the GS and replacing the EM locatable guide with the biopsy needle, illustrating the failure to deliver the needle in the lesion. GS, guiding sheath; AF, augmented fluoroscopy; CBCT, cone beam computed tomography; EM, electromagnetic tracking.

for bronchoscopy biopsy has led to greater bronchoscopist success in navigating biopsy instruments to smaller and more peripheral nodules with virtual bronchoscopy (7). However, the precurved delivery angle for each GS/needle combination restricts the range of delivery freedom, as demonstrated in the current study. In addition, when an EM tracker was exchanged with a stiffer biopsy instrument, the needle delivery angle decreased $2-59 \%$ depending on the instrument. Although only a single case, the clinical case report demonstrated ENB guided precurved catheter failure, while the steerable GS with CT-based AF image guidance successfully targeted the challenging lesion. However, the most acutely angled medial Edge catheter was not studied in the preclinical or clinical cases.
CBCT provides information for preprocedural planning and for assessment of needle delivery accuracy for biopsy success. In the case of intraprocedural atelectasis, a nondiagnostic biopsy, or airway deformation due to stiff scopes or needles, a CBCT may be acquired to assess the airway and fidelity to the original geometry and AF planning. Moreover, the issue of CT-to-body divergence can be addressed since navigational planning may be updated based on new imaging data (32). In the case of a failed biopsy identified during the procedure, intraoperative replanning of navigation is possible based on a CBCT (33). Combining preprocedural $\mathrm{CT}$ or $\mathrm{CBCT}$-based $\mathrm{AF}$ with intra-procedural CBCT for treatment planning maximized the use of both technologies $(13,14,26)$. Although CBCT-guided 

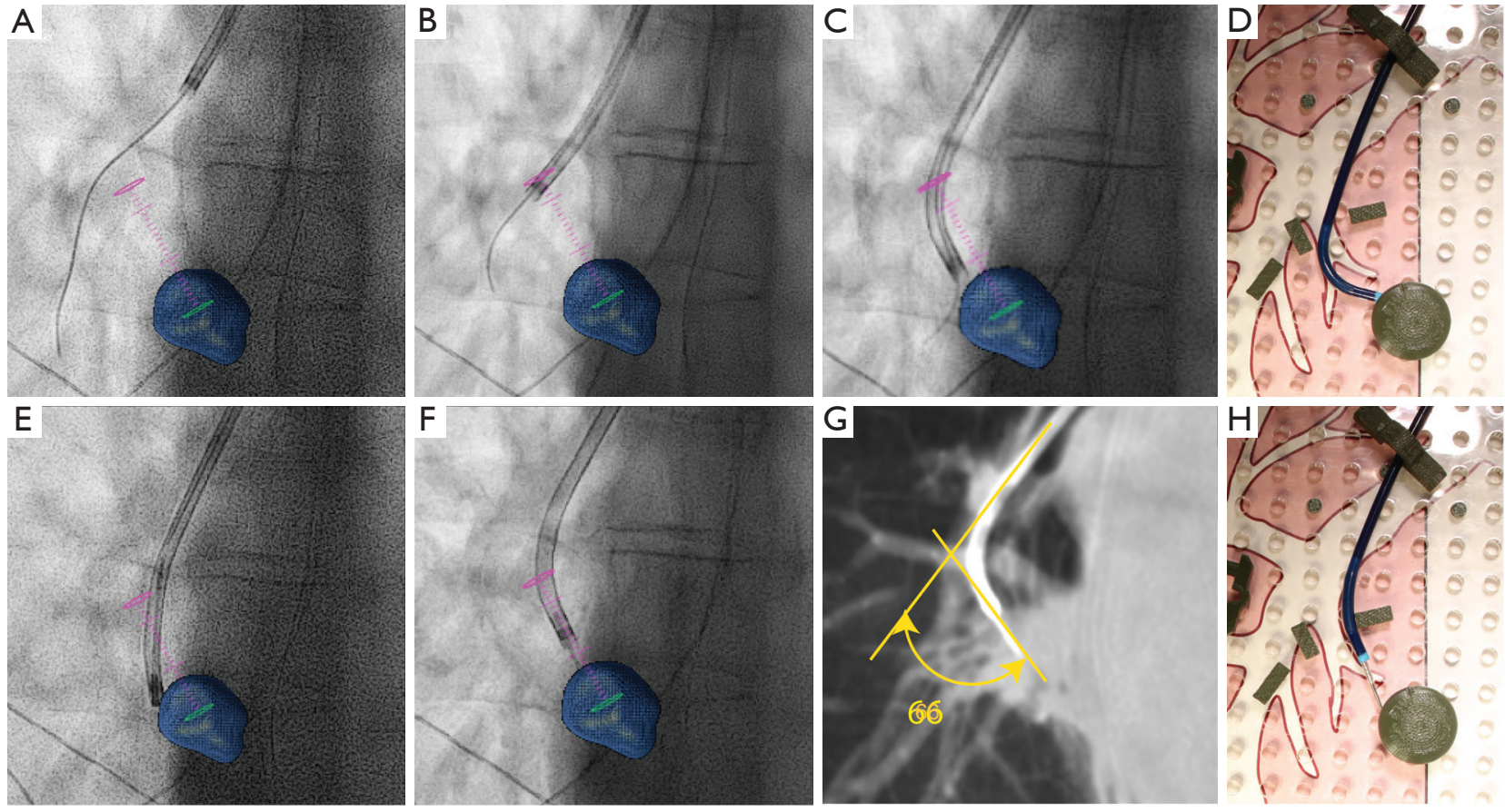

Figure 10 Navigation and biopsy of the central tumor guided by a steerable sheath under AF and CBCT image guidance. (A) The operator navigates the sheath through the airways over a guidewire. The segmented lesion (blue) and the guidance overlay on the fluoroscopic image are shown, including the planned airway exit point (purple circle), planned needle endpoint (teal circle), and needle trajectory (purple line). (B) Catheter positioned with the tip at the planned airway puncture site. (C) The sheath tip is deflected by rotating the catheter handle to gain the preferred angle towards the tumor. (D) Simulation of the steerable catheter with the tip flexed and pointing toward the lesion before the introduction of a biopsy instrument. (E) Wang needle is introduced, deflecting the DT tip to a straighter angle. (F) The operator deflects the DT angle to compensate for tip deformation, achieving the desired angle for insertion of the needle into the target. (G) A CBCT confirmed the position of the needle in the target. $(\mathrm{H})$ Simulation of the steerable catheter with the proximal tip deflected to point toward the lesion after the introduction of a biopsy needle, illustrating successful targeting of the lesion. DT, Destino twist; AF, augmented fluoroscopy; CBCT, cone beam computed tomography.

procedures have higher radiation exposure in comparison to EM-guided bronchoscopy procedures (20), radiation doses may be decreased by using optimized radiation dose settings for fluoroscopy and CBCT in CBCT-guided bronchoscopy procedures and adequate shielding (22).

Two endobronchial robotic navigation systems are currently under evaluation that could improve the diagnostic accuracy of peripheral lung biopsies $(32,34)$. Similar to the steerable GS, the primary advantage of the robotics systems is the improved steerability and tip stability of the GS when delivering the biopsy needle. However, accessibility, space limitations, cost, and required expertise pose challenges for this early technology. Conversely, steerable GS are readily available and may provide greater precision for biopsy of smaller targets while improving the capacity to target multiple regions to assess lung tumor heterogeneity.
Detection and management of pulmonary hemorrhage without the use of a bronchoscope remains a concern. However, percutaneous hemorrhage post-CT-TTNA occurs but rarely requires intervention (27). In addition, a bronchoscopy evaluation pre- or post- steerable GSguided biopsy may be required, in addition to EBUS guided mediastinal/hilar staging. Moreover, CT and CBCT detect vessels and hemorrhage, and bronchoscopic inspection of the airway pre- and post-biopsy may be conducted. In this study, the first two in vivo needle deliveries resulted in grade 1 and 2 pulmonary hemorrhage. CBCT was not acquired to confirm needle trajectory prior to the first needle delivery attempt. All subsequent needle deliveries utilized a confirmatory CBCT to evaluate the actual GS position and GS delivery angle and used updated AF views based on the updated $\mathrm{CBCT}$ acquisition to optimize the needle delivery 
prior to the first needle delivery attempt. This adapted workflow did not result in hemorrhage. These subsequent navigation and needle delivery tasks were also associated with reduced procedure time, fluoroscopy time, and the number of CBCT required. Moreover, the prototype AF software had additional features that enhanced AF workflow, including CBCT airway segmentation, navigation pathway trajectory analysis, and CT-CBCT registration and needle planning, compared to the commercial software AF.

In the future, incorporating GS deflection, the mitigation of deflection by use of steerable GS, and needle characteristics into treatment planning software to define the optimal planned needle delivery angle could further improve navigation planning and guidance as well as device selection. Extension of the work to study the properties of the biopsy instruments and their impact on targeting would be informative (33), as it has been shown that having a range of biopsy tools available increases the chance to provide a valid diagnostic outcome (35). Improved navigation software and device designs may advance interventions without a bronchoscope, while bronchoscopy may benefit from incorporating steerable GS and the use of CBCT during the procedures.

There were limitations to this study. The ex vivo lung did not have the normal, native configuration, and both the ex vivo and healthy swine lung have different tissue properties compared to human tumors or lung disease. While swine are an accepted pulmonary intervention model, the geometry and branching pattern of the swine airway differs from that of humans (25). The ex vivo and in vivo models required anatomical targets without performing tissue biopsy. The effect of fatigue and exposure to body temperature on device shape and performance was not evaluated in this study. In addition, there was a learning curve over the course of the study related to AF, the use of intraprocedural CBCT, and the behavior of the biopsy instruments. As shown in the case report, the results of this study are translatable to humans, but clinical studies with improved image guidance utilizing the novel AF prototype instead of the current AF software may further optimize steerable GS guided transbronchial needle delivery.

We have demonstrated the bench to bedside feasibility of the use of AF guidance and endovascular steerable GS without a bronchoscope, together with intraprocedural CBCT, to perform a biopsy in a lung lesion successfully. Furthermore, the preclinical comparison showed the advantages of using steerable GS, compared to precurved GS, in ensuring biopsy instruments can accurately target pulmonary lesions. The use of steerable GS may improve the diagnostic yield of transbronchial biopsy in settings with fluoroscopic and CBCT capability, whether in interventional radiology and bronchoscopy suites or hybrid operating rooms. However, a clinical trial is required to evaluate the safety and benefits of AF guidance software as well as the applications of endovascular steerable GS in the airway.

\section{Acknowledgments}

We would like to acknowledge Lucia Fonseca and William van der Sterren from Philips Healthcare for the development of the augmented fluoroscopy prototype software.

Funding: This study was supported by the Intramural Research Program of the National Institute of Health clinical center and Center for Interventional Oncology (NIH Z01 1ZID BC011242, and CL040015). NIH and Philips have a Cooperative Research and Development Agreement that provided support for this research.

\section{Footnote}

Reporting Checklist: The authors have completed the ARRIVE reporting checklist. Available at https://dx.doi. org/10.21037/tlcr-21-275

Data Sharing Statement: Available at https://dx.doi. org/10.21037/tlcr-21-275

Peer Review File: Available at https://dx.doi.org/10.21037/ tlcr-21-275

Conflicts of Interest: All authors have completed the ICMJE uniform disclosure form (available at https://dx.doi. org/10.21037/tlcr-21-275). NAV is an employee of Philips Research North America. Marco Verstege is an employee of Philips, Best, The Netherlands. NIH and Philips have a Cooperative Research and Development Agreement that may provide travel. NIH and Boston Scientific have a Cooperative Research and Development Agreement. Unrelated specifically to this work, Philips and NIH have licensing agreements for NIH patents, and Philips pays $\mathrm{NIH}$ royalties, who in turn pay BW royalties. NIH may have intellectual property or patents in the related field. The other authors have no conflicts of interest to declare.

Ethical Statement: The authors are accountable for all aspects of the work in ensuring that questions related 
to the accuracy or integrity of any part of the work are appropriately investigated and resolved. Experiments were performed under an animal study protocol (DRD 17-01) approved by the Institutional Animal Care and Use Committee in compliance with the Public Health Service (PHS) Policy on Humane Care and Use of Laboratory Animals (Policy). All procedures performed in studies involving human participants were in accordance with the ethical standards of the institution.

Open Access Statement: This is an Open Access article distributed in accordance with the Creative Commons Attribution-NonCommercial-NoDerivs 4.0 International License (CC BY-NC-ND 4.0), which permits the noncommercial replication and distribution of the article with the strict proviso that no changes or edits are made and the original work is properly cited (including links to both the formal publication through the relevant DOI and the license). See: https://creativecommons.org/licenses/by-nc-nd/4.0/.

\section{References}

1. Wang KP, Gonullu U, Baker R. Transbronchial Needle Aspiration Versus Transthoracic Needle Aspiration in the Diagnosis of Pulmonary Lesions. J Bronchology Interv Pulmonol 1994;1:199-204.

2. DiBardino DM, Yarmus LB, Semaan RW. Transthoracic needle biopsy of the lung. J Thorac Dis 2015;7:S304-16.

3. Zhang W, Chen S, Dong X, et al. Meta-analysis of the diagnostic yield and safety of electromagnetic navigation bronchoscopy for lung nodules. J Thorac Dis 2015;7:799-809.

4. Panchabhai TS, Mehta AC. Historical perspectives of bronchoscopy. Connecting the dots. Ann Am Thorac Soc 2015;12:631-41.

5. Wada H, Hirohashi K, Nakajima T, et al. Assessment of the new thin convex probe endobronchial ultrasound bronchoscope and the dedicated aspiration needle: a preliminary study in the porcine lung. J Bronchology Interv Pulmonol 2015;22:20-7.

6. Oki M, Saka H, Ando M, et al. Ultrathin Bronchoscopy with Multimodal Devices for Peripheral Pulmonary Lesions. A Randomized Trial. Am J Respir Crit Care Med 2015;192:468-76.

7. Folch EE, Pritchett MA, Nead MA, et al. Electromagnetic Navigation Bronchoscopy for Peripheral Pulmonary Lesions: One-Year Results of the Prospective, Multicenter NAVIGATE Study. J Thorac Oncol 2019;14:445-58.
8. Ost DE, Ernst A, Lei X, et al. Diagnostic Yield and Complications of Bronchoscopy for Peripheral Lung Lesions. Results of the AQuIRE Registry. Am J Respir Crit Care Med 2016;193:68-77.

9. Ali MS, Trick W, Mba BI, et al. Radial endobronchial ultrasound for the diagnosis of peripheral pulmonary lesions: A systematic review and meta-analysis. Respirology 2017;22:443-53.

10. Slavova-Azmanova NS, Lizama C, Johnson CE, et al. Impact of the introduction of EBUS on time to management decision, complications, and invasive modalities used to diagnose and stage lung cancer: a pragmatic pre-post study. BMC Cancer 2016;16:44.

11. Medford AR, Bennett JA, Free CM, et al. Endobronchial ultrasound-guided transbronchial needle aspiration (EBUS-TBNA): applications in chest disease. Respirology 2010;15:71-9.

12. Sainz Zuñiga PV, Vakil E, Molina S, et al. Sensitivity of Radial Endobronchial Ultrasound-Guided Bronchoscopy for Lung Cancer in Patients With Peripheral Pulmonary Lesions: An Updated Metaanalysis. Chest 2020;157:994-1011.

13. Silvestri GA, Bevill BT, Huang J, et al. An Evaluation of Diagnostic Yield From Bronchoscopy: The Impact of Clinical/Radiographic Factors, Procedure Type, and Degree of Suspicion for Cancer. Chest 2020;157:1656-64.

14. Pritchett MA. Prospective Analysis of a Novel Endobronchial Augmented Fluoroscopic Navigation System for Diagnosis of Peripheral Pulmonary Lesions. J Bronchology Interv Pulmonol 2021;28:107-15.

15. Cicenia J, Bhadra K, Sethi S, et al. Augmented Fluoroscopy: A New and Novel Navigation Platform for Peripheral Bronchoscopy. J Bronchology Interv Pulmonol 2021;28:116-23.

16. Ali EAA, Takizawa H, Kawakita N, et al. Transbronchial Biopsy Using an Ultrathin Bronchoscope Guided by Cone-Beam Computed Tomography and Virtual Bronchoscopic Navigation in the Diagnosis of Pulmonary Nodules. Respiration 2019;98:321-8.

17. Casal RF, Sarkiss M, Jones AK, et al. Cone beam computed tomography-guided thin/ultrathin bronchoscopy for diagnosis of peripheral lung nodules: a prospective pilot study. J Thorac Dis 2018;10:6950-9.

18. Hohenforst-Schmidt W, Zarogoulidis P, Vogl T, et al. Cone Beam Computertomography (CBCT) in Interventional Chest Medicine - High Feasibility for Endobronchial Realtime Navigation. J Cancer 2014;5:231-41.

19. Pritchett MA, Schampaert S, de Groot JAH, et al. Cone- 
Beam CT With Augmented Fluoroscopy Combined With Electromagnetic Navigation Bronchoscopy for Biopsy of Pulmonary Nodules. J Bronchology Interv Pulmonol 2018;25:274-82.

20. Verhoeven RLJ, Fütterer JJ, Hoefsloot W, et al. Cone-Beam CT Image Guidance With and Without Electromagnetic Navigation Bronchoscopy for Biopsy of Peripheral Pulmonary Lesions. J Bronchology Interv Pulmonol 2021;28:60-9.

21. Bydlon TM, Langhout GC, Lalezari F, et al. Optimal endobronchial tool sizes for targeting lung lesions based on 3D modeling. PLoS One 2017;12:e0189963.

22. de Ruiter QMB, Karanian JW, Bakhutashvili I, et al. Endobronchial Navigation Guided by Cone-Beam CTBased Augmented Fluoroscopy without a Bronchoscope: Feasibility Study in Phantom and Swine. J Vasc Interv Radiol 2020;31:2122-31.

23. O'Shea C, Khan KA, Tugwell J, et al. Loss of flexion during bronchoscopy: a physical experiment and case study of commercially available systems. Lung Cancer Manag 2017;6:109-18.

24. Oki M, Saka H, Kogure Y. Transesophageal needle aspiration using a third-generation Olympus ultrasound bronchoscope for subaortic lesions: a report of two cases. Transl Lung Cancer Res 2019;8:1152-6.

25. Castro MGB, Varble NA, Yung RC, et al. In vivo Characterization of the Swine Airway Morphometry and Motion Based on Computed Tomographic Imaging During Respiration. J Biomech Eng 2020;142:121009.

26. Amin MB, Edge S, Greene F, et al. AJCC Cancer Staging Manual (8th edition). Springer International Publishing: American Joint Commission on Cancer. 2017.

27. Tai R, Dunne RM, Trotman-Dickenson B, et al. Frequency and Severity of Pulmonary Hemorrhage in Patients Undergoing Percutaneous CT-guided Transthoracic Lung
Biopsy: Single-Institution Experience of 1175 Cases. Radiology 2016;279:287-96.

28. Goussard P, Pohunek P, Eber E, et al. Pediatric bronchoscopy: recent advances and clinical challenges. Expert Rev Respir Med 2021;15:453-75.

29. Makaloski V, Tsilimparis N, Rohlffs F, et al. Use of a Steerable Sheath for Retrograde Access to Antegrade Branches in Branched Stent-Graft Repair of Complex Aortic Aneurysms. J Endovasc Ther 2018;25:566-70.

30. Deyell MW, Wen G, Laksman Z, et al. The impact of steerable sheaths on unblinded contact force during catheter ablation for atrial fibrillation. J Interv Card Electrophysiol 2020;57:417-24.

31. Ullah W, Hunter RJ, McLean A, et al. Impact of steerable sheaths on contact forces and reconnection sites in ablation for persistent atrial fibrillation. J Cardiovasc Electrophysiol 2015;26:266-73.

32. Fielding DIK, Bashirzadeh F, Son JH, et al. First Human Use of a New Robotic-Assisted Fiber Optic Sensing Navigation System for Small Peripheral Pulmonary Nodules. Respiration 2019;98:142-50.

33. Li P, Yang Z, Jiang S. Needle-tissue interactive mechanism and steering control in image-guided robot-assisted minimally invasive surgery: a review. Med Biol Eng Comput 2018;56:931-49.

34. Chen AC, Pastis NJ Jr, Mahajan AK, et al. Robotic Bronchoscopy for Peripheral Pulmonary Lesions: A Multicenter Pilot and Feasibility Study (BENEFIT). Chest 2021;159:845-52.

35. Gildea TR, Folch EE, Khandhar SJ, et al. The Impact of Biopsy Tool Choice and Rapid On-Site Evaluation on Diagnostic Accuracy for Malignant Lesions in the Prospective: Multicenter NAVIGATE Study. J Bronchology Interv Pulmonol 2021;28:174-83.
Cite this article as: de Ruiter QMB, Fontana JR, Pritchard WF, Mauda-Havakuk M, Bakhutashvili I, Esparza-Trujillo JA, Varble NA, Verstege M, Xu S, Seifabadi R, Browning RF, Wood BJ, Karanian JW. Endovascular steerable and endobronchial precurved guiding sheaths for transbronchial needle delivery under augmented fluoroscopy and cone beam CT image guidance. Transl Lung Cancer Res 2021;10(8):3627-3644. doi: $10.21037 /$ tlcr-21-275 


\section{Bench model description}

A bench model was developed to simulate needle delivery and measure GS deflection. The plexiglass sheet had a laser-cut grid of $1 / 8$ " holes at $1 \mathrm{~cm}$ intervals, 3D printed clips fitted to the holes to guide the GS shaft on the grid, and 3D printed 2 $\mathrm{cm}$ diameter spherical targets. Images of the GS were captured using a digital single-lens reflex camera perpendicular to the platform, and GS deflection and angles and errors were digitally measured with ImageJ (NIH, Bethesda, MD, USA).

\section{Ex vivo lung model description}

GS navigation and needle deliveries were conducted in an inflatable plasticized swine lung (BioQuest@ Inflatable Lung Kit, Nasco, Fort Atkinson, WI). Gel water beads, a cross-linked super absorbent crystal polymer (Magic Beadz, Sanavet Laboratories LLC, US), absorb liquid and swell in size to an average solid sphere size of $6 \pm 2 \mathrm{~mm}$ after injection in the lung $(\mathrm{n}=5)$. Beads were injected in the lung transpleurally through a $6 \mathrm{Fr}$ introducer sheath to serve as surrogate lung targets. The puncture sites were sealed with a drop of liquid bandage. The lung was placed in a sealed container and mechanically ventilated using a vacuum pump connected to the container. GS navigation and needle delivery to each target were performed with CBCT-based AF image guidance.

\section{Swine management and anesthesia details}

Anesthesia: Animals were sedated with intramuscular ketamine $(25 \mathrm{mg} / \mathrm{kg})$, midazolam $(0.5 \mathrm{mg} / \mathrm{kg})$, and glycopyrrolate $(0.01 \mathrm{mg} / \mathrm{kg})$ and anesthetized with Propofol $(1 \mathrm{mg} / \mathrm{kg} \mathrm{IV})$. Animals were intubated, maintained under general anesthesia with isoflurane, 1-5\% (Isoflo, Abbott Animal Health; North Chicago, IL), and mechanically ventilated with breathing temporarily suspended during CT or CBCT acquisition. At the conclusion of the study, euthanasia was performed under general anesthesia by intravenous administration of Beuthanasia-D (pentobarbital sodium $390 \mathrm{mg} / \mathrm{mL}$ and phenytoin sodium $50 \mathrm{mg} / \mathrm{mL}$ ).

Swine Housing conditions: The Division of Veterinary Resources (DVR) facility set point for swine is $72^{\circ} \mathrm{F}+/-3^{\circ} \mathrm{F}$. The DVR facility set point for humidity is $50 \%$. Animals have outdoor access when temperatures are $>32^{*} \mathrm{~F}$ and $<90^{*} \mathrm{~F}$.

Animal Enrichment: Pigs are socially housed and provided with toys to encourage normal behaviors. Animals are provided with fresh vegetables and foraging materials. All animals were enriched and housed in groups prior to experimentation.

Animal Health Status: Animals come from a closed colony and the herd is free of PRRS, PRV, Brucellosis, Myocoplasma hypopneumoniae, and APP. Animals are vaccinated with an autogenous vaccine consisting of Streptococcus Suis, Mycoplasma Hyorhinis, Mycoplasma Hyosynoviae, and Haemophilus Parasuis, in addition to commercial vaccinations for swine influenza, Bordetella bronciseptica, porcine circovirus, Erysipelas rhusiophathiae and Salmonella Choleraesuis-Typhimurium.

Diet: Ziegler NIH Swine 2004-2. 